Int. J. Dev. Biol. 59: 497-503 (2015)

doi: $10.1387 / \mathrm{ijdb} .150124 \mathrm{sf}$

\title{
External ear microRNA expression profiles during mouse development
}

\author{
LEDA TORRES ${ }^{1}$, ULISES JUÁREZ ${ }^{1,2}$, LAURA GARCÍA ${ }^{3,4}$, JUAN MIRANDA-RíOS ${ }^{4}$ and SARA FRIAS*,1,5 \\ ${ }^{1}$ Laboratorio de Citogenética, Depto. de Investigación en Genética Humana, Instituto Nacional de Pediatría, ${ }^{2}$ Posgrado \\ en Ciencias Biológicas, Universidad Nacional Autónoma de México, ${ }^{3}$ Posgrado en Ciencias Biomédicas, Universidad \\ Nacional Autónoma de México, ${ }^{4}$ Unidad de Genética de la Nutrición, Depto. Biología Molecular y Biotecnología, Instituto \\ de Investigaciones Biomédicas, Universidad Nacional Autónoma de México e Instituto Nacional de Pediatría and ${ }^{5}$ Unidad \\ de Genética de la Nutrición. Depto. Medicina Genómica y Toxicología Ambiental, Instituto de Investigaciones Biomédicas, \\ Universidad Nacional Autónoma de México e Instituto Nacional de Pediatría, México DF, México
}

\begin{abstract}
MicroRNAs (miRNAs) comprise a class of approximately 22 nucleotide regulatory noncoding RNAs that play several roles in diverse biological processes. Recent reports suggest that embryonic development in mammals is accompanied by dynamic changes in miRNA expression; however, there is no information regarding the role of miRNAs in the development of the external ear. The aim of this study was to determine the stage-specific expression of miRNAs during mouse external ear development in order to identify potentially implicated miRNAs along with their possible targets. miRNA expression profiles from fetal mice pinnae and back skin tissues at 13.5 dpc and $14.5 \mathrm{dpc}$ were obtained using an Affymetrix GeneChip miRNA 3.0 array. Biological triplicates for both tissues, each collected from a litter averaging 16 fetuses, were analyzed. The results were analyzed with Affymetrix's Transcriptome Analysis Console software to identify differentially expressed miRNAs. We observed differential expression of 40 miRNAs including some predicted to target genes implicated in external ear development, such as mmu-miR-10a, a miRNA known to modulate Hoxa1 mRNA levels, and mmu-miR-200c and mmu-miR-205. To our knowledge, this is the first miRNA expression profiling study of external ear development in mammals. These data could set the basis to understand the implications of miRNAs in normal external ear development
\end{abstract}

KEY WORDS: miRNA, pinnae, auricle, microtia, GEO (GSE64945)

In mammals, the outer ear is formed by the ear pinna (i.e., the auricle and the external ear), the external acoustic meatus (i.e., the ear canal), and the outer layer of the tympanic membrane (i.e., the eardrum). The outer ear develops as a result of complex tissue interactions between ectoderm and mesoderm during embryogenesis. Outer ear development is driven by the mesenchyme of the first and second pharyngeal arches and is controlled, at least in part, by the genes that determine the first and the second pharyngeal arch identity. The auricle is formed from six protuberances in the first and the second arches known as the auricular hillocks (Jones and Chuan, 1934; Mallo and Gridley, 1996). In mice, several genes involved in external ear development have been identified, including Hoxa2, Hoxa1, Hoxb1, Prrx1, Dlx1, Dlx5, Six1, Six4, Sall1, Tbx1, Bmp5, Fgf3, Fgf8, Fgf10 and Eya1. The genetic and cellular mechanisms underlying normal morphogenesis of the external ear are incompletely understood (Minoux et al., 2013; Luquetti et al., 2012). Recent reports suggest that embryonic development in mammals is accompanied by dynamic changes in the expression of an extensive class of regulatory molecules termed miRNAs (Lau et al., 2001; Berezikov, 2011); therefore, these molecules could be influencing the external ear development process.

miRNAs are small (approximately 22 nucleotide long), highly conserved regulatory molecules that modulate gene expression, mainly by targeting mRNAs for translational repression or degradation (Lau et al., 2001; Berezikov, 2011). Since their discovery, these non-coding RNA molecules have been shown to play important roles in several biological processes, including vertebrate develop-

Abbreviations used in this paper: dpc, days post coitum; mmu, Mus musculus.

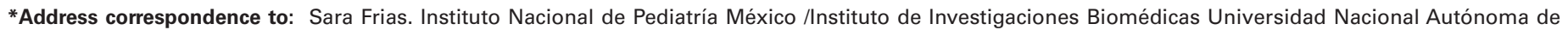
México. Insurgentes Sur 3700-C, CP 04530, Col. Insurgentes-Cuicuilco, Mexico D.F., México. Tel: +52-55-1084-5533.

E-mail: sarafrias@biomedicas.unam.mx; sarafrias@yahoo.com
}

Accepted: 2 December 2015.

ISSN: Online 1696-3547, Print 0214-6282 
ment (Gavalas et al., 1998; Petri et al., 2014; Pasquinelli, 2012).

The study of miRNAs is a novel field, and little is known about their role in organogenesis (the development of organs or tissues) or the mechanisms that regulate their expression and function (Pasquinelli, 2012). However, there have been several mice studies that explored the role of miRNAs in the development of heart, retina, brain and inner ear. These studies have shown a particular spatiotemporal pattern of miRNAs expression, where specific sets of miRNAs are expressed in the developmental processes of each structure (Petri et al., 2014; Sacheli et al., 2009).

In the mouse, the study of miRNAs in the inner ear has shown temporal and spatial expression of the miR-96/-182/-183 cluster that correlates with the inner ear's functional maturation (Sacheli et al., 2009). However, the miRNA expression profile during external ear development has not been assayed.

The purpose of this study was to determine the stage-specific expression of miRNAs during external ear development in mice under normal conditions and to identify the miRNAs along with their possible targets.

\section{Results and Discussion}

We describe the expression pattern of miRNAs involved in the development of the external ear in mice at two different stages of development; 13.5 days post coitum (dpc) and $14.5 \mathrm{dpc}$, using GeneChip miRNA 3.0 arrays from Affymetrix. We compared the expression profiles from pinnae and back skin tissues and observed, as expected, that there are distinct patterns of miRNA expression between the tissues and the stages of development. The differentially expressed miRNAs between pinnae and back skin tissues at $13.5 \mathrm{dpc}$ and $14.5 \mathrm{dpc}$ are shown as heat maps in Figs 1 and 2, respectively.

Spatial expression pattern showed by in situhybridization of mmu-miR-10a, mmu-miR-205 and mmu-miR-200c at 13.5 and $14.5 \mathrm{dpc}$, confirmed the results observed by microarrays. We observed signal of mmu-miR-10a at both stages analyzed in the dorsal region and in the bones of anterior limbs, and almost undetectable signal in pinnae, the signal for mmu-miR-200c and mmu-miR-205 was present in lips and nose, as previously reported (Warner et al., 2014) and in pinnae, with high levels of mmu-miR-205 and low levels in the case of mmu-miR200c at both stages (Fig. 3).

We performed real-time quantitative RT-PCR analysis in the interest to know if mRNAs of Hoxa1, Hoxa2, Tob1, Eya1 and Six1 were present in pinnae or skin at the stages studied. The relative expression levels of mRNAs

Fig. 1. Heat map of miRNA expression at $13.5 \mathrm{dpc}$, showing differentially expressed miRNAs between pinnae and back skin tissues. Hierarchical clusters of 25 miRNA genes were found to be significantly differentially expressed (adjusted p value $<0.05$ ) in pinnae compared to back skin controls. Each row of the hierarchical clusters represents an individual miRNA and each column represents one biological replica of either pinnae tissue (1) or skin tissue (2). The scale represents the gene expression intensity (log2 scale ranges between -1.5 and 1.5). Red color indicates an increase in miRNA gene expression (relative to the other expression measurements in the same row) and green indicates a decrease. in relation to the Actb reference gene are presented in Fig. 4. We detected mRNA of the five genes studied in both tissues.

The microarrays analysis showed in fetal mice of $13.5 \mathrm{dpc}, 25$ different mmu-miRNAs differentially expressed in the pinnae with 11 of the mmu-miRNAs presenting elevated expression levels and 14 with lowered expression levels (Table 1). At $14.5 \mathrm{dpc}, 31$ different mmu-miRNAs showed differential expression in the pinnae with 14 mmu-miRNAs displaying elevated levels of expression while 17 exhibited decreased levels of expression (Table 2). We observed that mmu-miR-429, mmu-miR-375, mmu-miR-200a, mmu-miR-708, mmu-miR-27b-5p, mmu-miR-23b and mmu-miR-24 were highly expressed in both stages. These miRNAs have several possible targets according to the consulted databases; some of them (like Eya1, Hoxa2 and Six4) are known to be related to external ear development. However, their interactions have not yet been corroborated.

Moreover, at both stages we detected in the microarrays some miRNAs with low expression levels, such as mmu-miR-7a-2-3p, mmu-miR-135a-2-3p, mmu-miR-10b, mmu-miR-615-3p, mmumiR-9-3p, mmu-miR-219-3p and mmu-miR-10a. The low levels of expression of mmu-miR-10a in pinnae were also seen in in situ hybridization analysis at both stages analyzed, in detail the pinnae has significantly lower signal of mmu-miR-10a in the outline of the ear canal, as compared with back skin; note the cartilaginous region without signal. (Fig. $3 \mathrm{C}$ and $3 \mathrm{E}$ ). The inquiry for possible targets of these miRNAs showed several genes. Of note is Hoxa1, a gene that participates in the development of the external ear that is a mmu-miR-10a target. In mice, the mmu-miR-10a gene-coding region is located at the $11 \mathrm{qD}$ locus within the $\operatorname{Hox} B$ gene cluster.

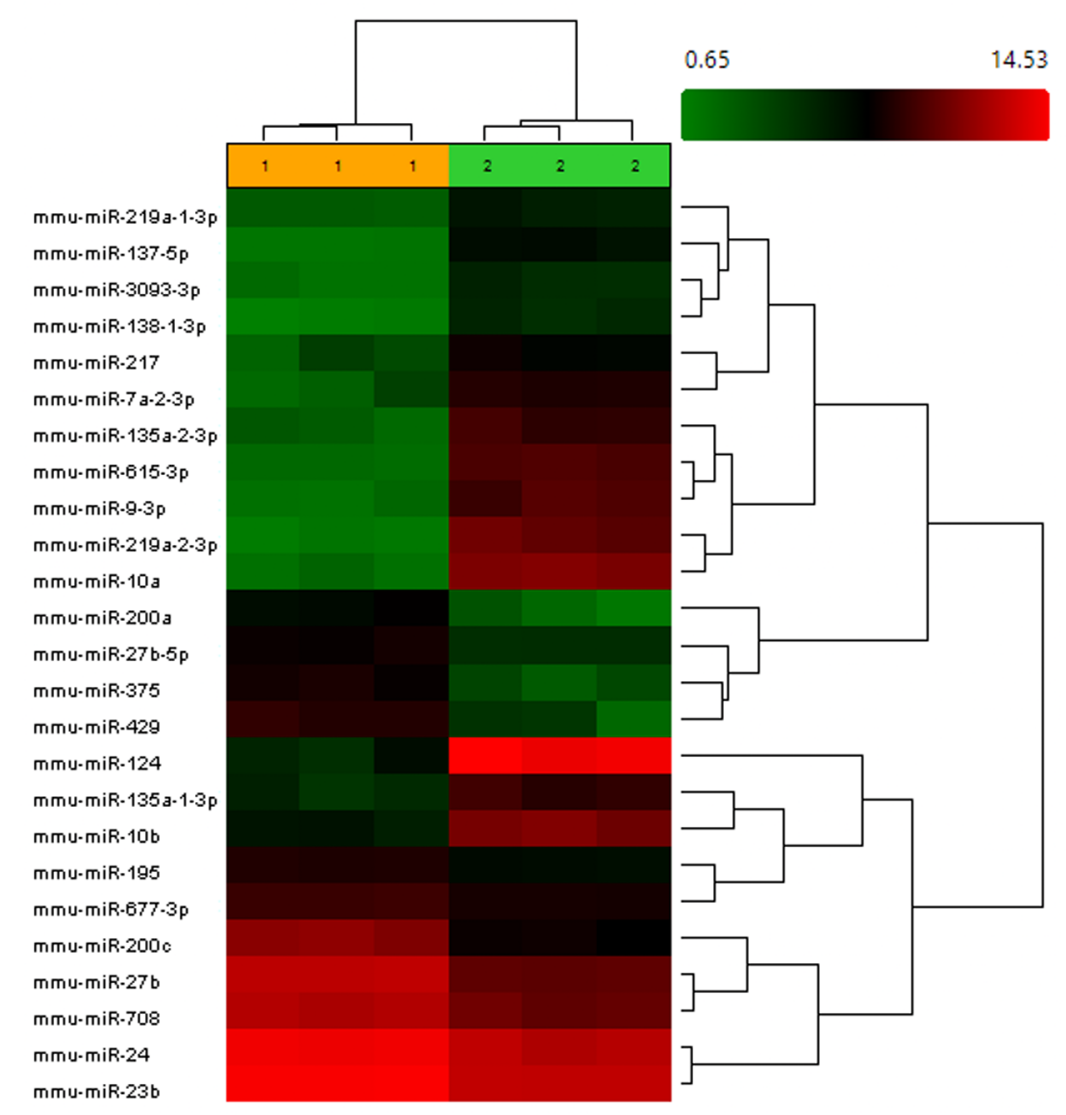


TABLE 1

THE miRNAs DIFFERENTIALLY EXPRESSED IN PINNAE VERSUS SKIN BACK TISSUES AT 13.5 DPC

\begin{tabular}{llcc} 
mmu-miRnA & Sequence (5' $^{\prime}$-3') & $\begin{array}{c}\text { Length } \\
\text { (nt) }\end{array}$ & $\begin{array}{c}\text { Fold } \\
\text { change }\end{array}$ \\
\hline mmu-miR-200c & UAAUACUGCCGGGUAAUGAUGGA & 23 & 3.18 \\
mmu-miR-429 & UAAUACUGUCUGGUAAUGCCGU & 22 & 2.80 \\
mmu-miR-27b & UUCACAGUGGCUAAGUUCUGC & 22 & 2.52 \\
mmu-miR-375 & UUUGUUCGUUCGGCUCGCGUGA & 22 & 2.43 \\
mmu-miR-200a & UAACACUGUCUGGUAACGAUGU & 22 & 2.09 \\
mmu-miR-708 & AAGGAGCUUACAAUCUAGCUGGG & 23 & 1.92 \\
mmu-miR-27b-5p & AGAGCUUAGCUGAUUGGUGAAC & 22 & 1.85 \\
mmu-miR-23b & AUCACAUUGCCAGGGAUUACC & 21 & 1.57 \\
mmu-miR-24 & UGGCUCAGUUCAGCAGGAACAG & 22 & 1.56 \\
mmu-miR-195 & UAGCAGCACAGAAAUAUUGGC & 21 & 1.16 \\
mmu-miR-677-3p & GAAGCCAGAUGCCGUUCCUGAGAAGG & 26 & 0.86 \\
mmu-miR-3093-3p & UGUGGACACCGUGGGAGGUUGG & 22 & -1.06 \\
mmu-miR-138-1-3p & CGGCUACUUCACAACACCAGGG & 22 & -1.17 \\
mmu-miR-219a-1-3p & AGAGUUGCGUCUGGACGUCCCG & 22 & -1.29 \\
mmu-miR-137-5p & ACGGGUAUUCUUGGGUGGAUAAU & 23 & -1.95 \\
mmu-miR-217 & UACUGCAUCAGGAACUGACUGGA & 23 & -2.07 \\
mmu-miR-135a-1-3p & UAUAGGGAUUGGAGCCGUGGCG & 22 & -2.61 \\
mmu-miR-7a-2-3p & CAACAAGUCCCAGUCUGCCACA & 22 & -2.91 \\
mmu-miR-135a-2-3p & UGUAGGGAUGGAAGCCAUGAA & 21 & -3.52 \\
mmu-miR-10b & UACCCUGUAGAACCGAAUUUGUG & 23 & -3.75 \\
mmu-miR-615-3p & UCCGAGCCUGGGUCUCCCUCUU & 22 & -4.17 \\
mmu-miR-9-3p & AUAAAGCUAGAUAACCGAAAGU & 22 & -4.23 \\
mmu-miR-219a-2-3p & AGAAUUGUGGCUGGACAUCUGU & 22 & -4.96 \\
mmu-miR-10a & UACCCUGUAGAUCCGAAUUUGUG & 23 & -5.49 \\
\hline & UAAGGCACGCGGUGAAUGCC & 20 & -7.40 \\
\hline
\end{tabular}

nt: nucleotides
It has been reported that in mouse embryo, miR-10a expression follows a HoxB-like expression pattern with a common regulatory element (Tanzer et al., 2005). According to previous luciferase activity studies, it was suggested that miR-10a could be an inhibitor of the HOXA1 expression in megakaryocytic differentiation (Garzon et al., 2006). It seems that when mmu-miR-10a expression is low, as shown in our results, Hoxa1 mRNA levels will not be downregulated, our results demonstrate that Hoxa1 mRNA levels in pinnae was higher than that observed in back skin. Moreover, a report of a Hoxa 1 null mouse model revealed that the deficient mice present auricle hypoplasia (Gavalas et al., 1998). These findings support that miR-10a has an important role during pinnae development in mammals, most likely by modulating the Hoxa 1 expression.

A spatiotemporal pattern of miRNA expression, similar to what has been described in other tissues, was found in the external ear. Some of the detected miRNAs changed their expression levels from one stage to the other and from one site to the other (Tables 1 and 2). For example, mmu-miR-200c was expressed only at $13.5 \mathrm{dpc}$, while mmu-miR-205 was expressed at $14.5 \mathrm{dpc}$.

We detected mmu-miR-200c and mmu-miR-205 expression in the pinnae tissues of healthy mice (Fig. 3). This correlates with the reported findings in human patients with microtia where hsa-miR-200c and hsa-miR-205 have reduced expression in the pinnae (Li et al., 2013). Possible targets of mmu-miR-200c and mmu-miR-205 are the mRNAs of Six1 and Eya1. In mouse, Eya1 is expressed during outer and middle ear morphogenesis (13.5 dpc) the interaction between Eya1 and Six 1 is crucial for the morphogenesis of the cochlea and the posterior ampulla during inner

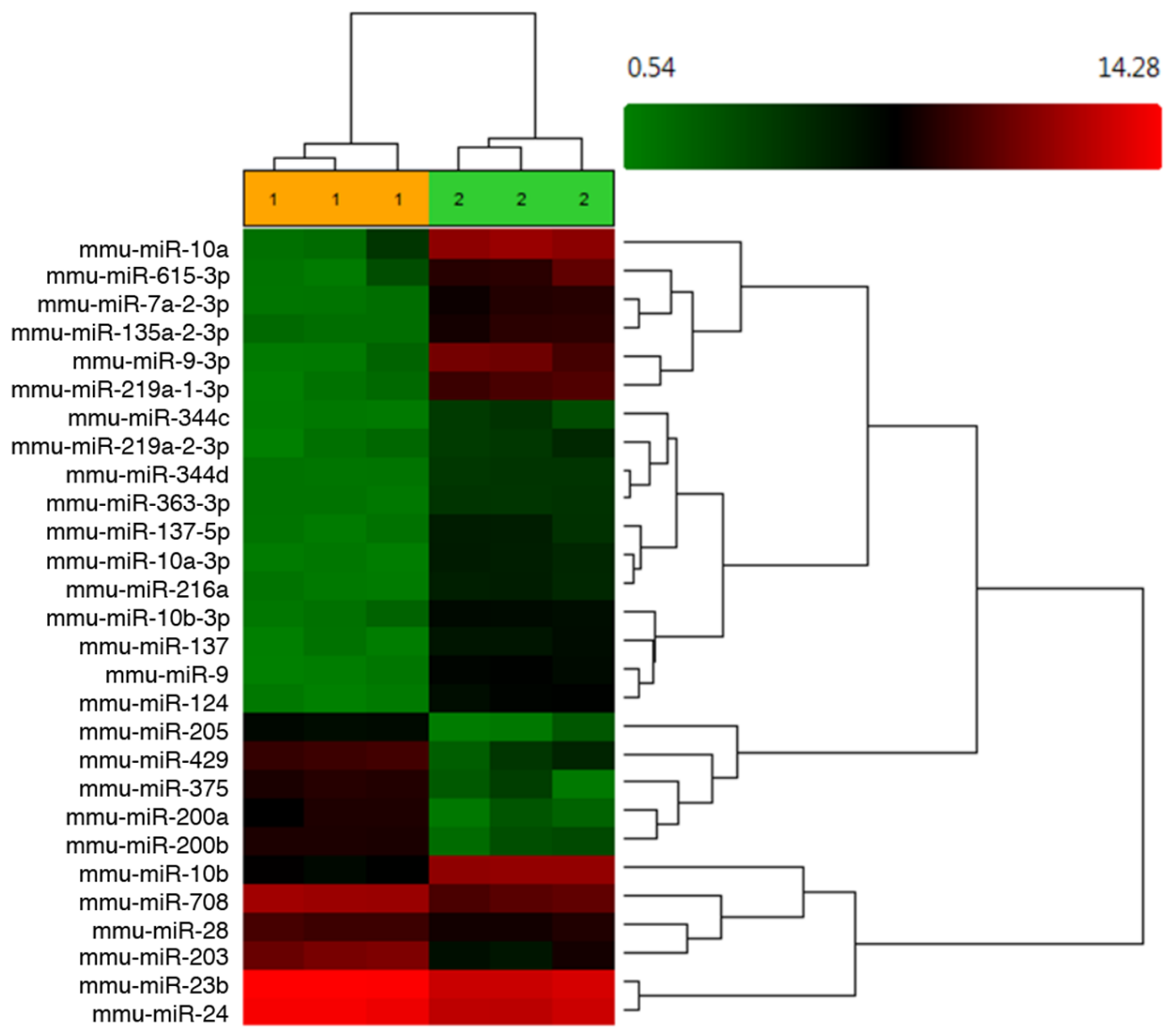

Fig. 2. Heat map of miRNA expression at $14.5 \mathrm{dpc}$, showing differentially expressed miRNAs between pinnae and back skin tissues. Hierarchical clusters of 31 miRNA genes were found to be significantly differentially expressed (adjusted $p$ value $<0.05$ ) in pinnae compared to back skin controls. Each row of the hierarchical clusters represents an individual miRNA and each column represents one biological replica of either pinnae tissue (1) or skin tissue (2). The scale represents the gene expression intensity (log2 scale ranges between -1.5 and 1.5). Red color indicates an increase in miRNA gene expression (relative to the other expression measurements in the same row) and green indicates a decrease. 


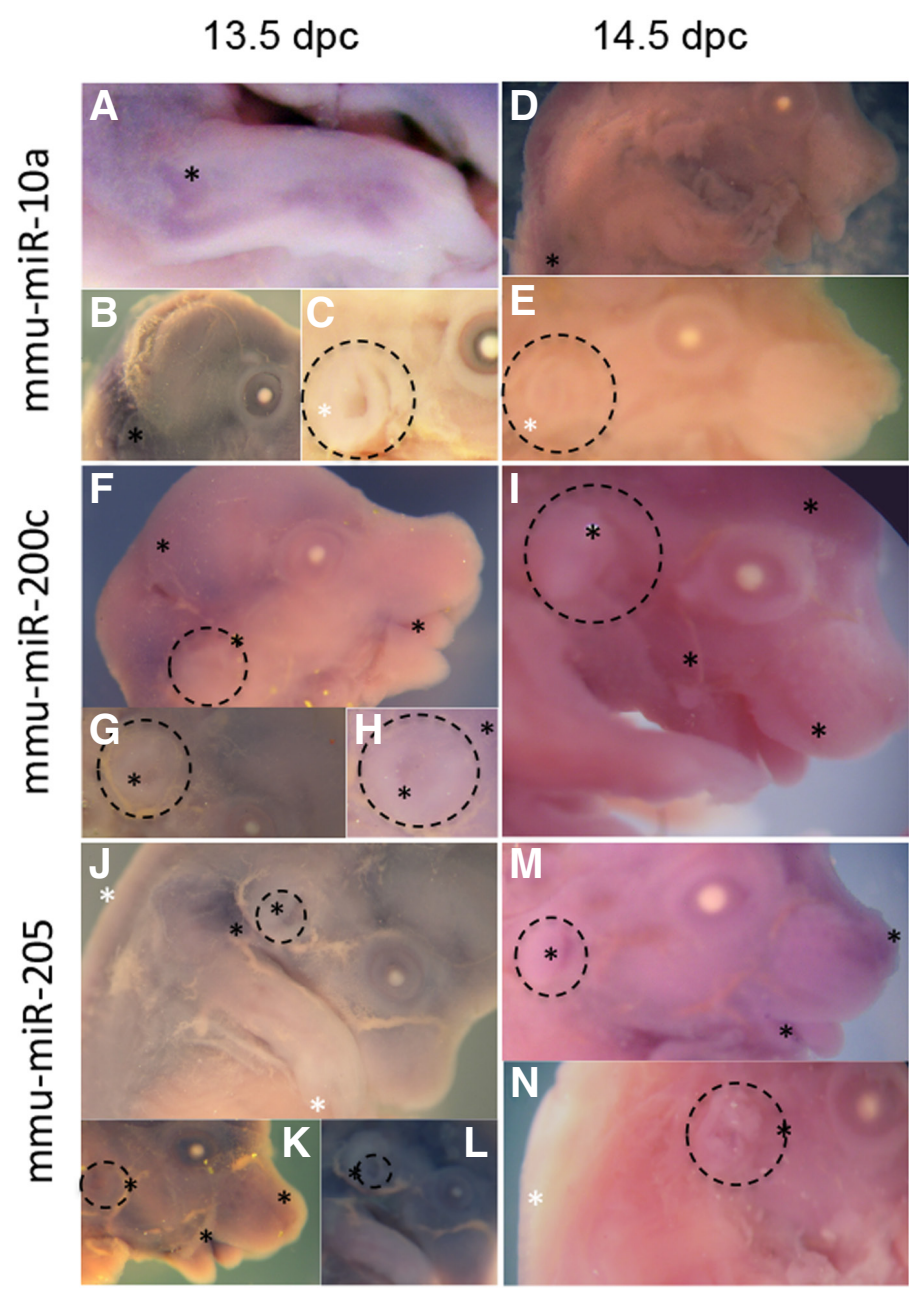

Fig. 3. miRNA in situ hybridization (ISH) at $\mathbf{1 3 . 5}$ and $\mathbf{1 4 . 5} \mathrm{dpc}$. Black (*) marks indicate positive signal; white $\left({ }^{*}\right)$ marks indicate absence of signal. Dashed circle surrounds pinna region. (A-C) ISH of mmu-miR-10a at 13.5 $d p c ;(\mathbf{D}, \mathbf{E})$ ISH of mmu-miR-10a at $14.5 \mathrm{dpc}$; (F-H) ISH of mmu-miR-200c at $13.5 \mathrm{dpc}$; (I) ISH of mmu-miR-200c at $14.5 \mathrm{dpc}$; (J-L) ISH of mmu-miR-205 at $13.5 \mathrm{dpc}$ and (M,N) ISH of mmu-miR-205 at $14.5 \mathrm{dpc}$. (A) Signal of mmu-miR-10a is present in the bone of anterior limbs. (B) Presence of mmu-miR-10a in the back skin, according with the microarrays results. (C) Pinna with significantly lower signal of mmu-miR-10a as compared with back skin. (D) Lateral view of fetus with strong mmu-miR-10a expression in the back skin. (E) Undetectable expression of mmu-miR-10a in pinna. (F) Presence of mmu-miR-200c in head and face 13.5 dpc, heavy mark in lips commissure, and light signal in skin surrounding pinnae. (G) and (H) Pinna closer view with detectable stain for mmu-miR-200c. (I) Head and face $14.5 \mathrm{dpc}$ with mmu-miR-200c detection, positive signal was observed in all face and head, including the pinna. (J) Detectable signal of mmu-miR-205 in pinna and surrounding skin, and undetectable signal in anterior limbs and back skin. (K) Nose, lips commissure and pinna with positive signal. (L) Closer view of pinna showing mmu-miR-205 expression. (M) mmu-miR-205 is present in pinna, lips and nose. (N) mmu-miR-205 is almost absent in back skin and present in the pinna area.

ear development. (Kalatzis et al., 1998; Zheng et al., 2003). In addition, it has been observed that mutations in the human genes EYA1, SIX1 and SIX5, cause the autosomal dominant syndrome Branchio-Oto-Renal, an early developmental defect characterized by varying combinations of branchial (fistulas, sinuses, and cysts), outer, middle and inner ear, as well as renal anomalies. (Song et al., 2013). The RT-PCR analysis confirms the presence of mRNA of Six1 and Eya1, both possible targets of mmu-miR-200c and $\mathrm{mmu}-\mathrm{miR}-205$. All together, these findings suggest that miR-200c and miR-205 have an important role during normal external ear development in mammals.

Furthermore, we observed in the pinnae tissues that mmumiR-124 expression levels changed between the two analyzed stages. This miRNA remains low during $13.5 \mathrm{dpc}$ and rises at 14.5 $\mathrm{dpc}$, indicating that its activity is required in this stage. Recent reports have implicated this miRNA in neuronal development and aging processes (Neo et al., 2014). A miR-124 knockout mouse model resulted in clear reduction of mature miR-124 levels that led to defective neuronal survival and axonal outgrowth, along with smaller brain size (Sanuki et al., 2011). The participation of miR-124 in the development of the external ear has not been reported. However, mmu-miR-124 is predicted to have several putative target genes implicated in external ear development, such as Six4, DIx5, Eya1 and Prrx1. Although none of these genes have been validated as direct targets of mmu-miR-124, these findings suggest that mmumiR-124 might contribute to external ear development.

Moreover, bioinformatics analyses of putative mRNA targets for differentially expressed miRNAs, allowed us to propose nine new candidate genes (Zeb1, Zeb2, Tob1, Snai1, Smad5, Runx1,

TABLE 2

\section{THE mIRNAS DIFFERENTIALLY EXPRESSED IN PINNAE VERSUS SKIN BACK TISSUES AT 14.5 DPC}

\begin{tabular}{|c|c|c|c|}
\hline mmu-miRNA & Sequence $\left(5^{\prime}-3^{\prime}\right)$ & $\begin{array}{c}\text { Length } \\
\text { (nt) }\end{array}$ & $\begin{array}{c}\text { Fold } \\
\text { change }\end{array}$ \\
\hline mmu-miR-205 & UCCUUCAUUCCACCGGAGUCUG & 22 & 5.92 \\
\hline mmu-miR-203 & GUGAAAUGUUUAGGACCACUAG & 22 & 3.76 \\
\hline mmu-miR-429 & UAAUACUGUCUGGUAAUGCCGU & 22 & 3.37 \\
\hline mmu-miR-200a & UAACACUGUCUGGUAACGAUGU & 22 & 2.74 \\
\hline mmu-miR-200b & UAAUACUGCCUGGUAAUGAUGA & 22 & 2.73 \\
\hline mmu-miR-375 & UUUGUUCGUUCGGCUCGCGUGA & 22 & 2.63 \\
\hline mmu-miR-205-3p & GAUUUCAGUGGAGUGAAGCUCA & 22 & 2.02 \\
\hline mmu-miR-708 & AAGGAGCUUACAAUCUAGCUGGG & 23 & 1.92 \\
\hline mmu-miR-27b-5p & AGAGCUUAGCUGAUUGGUGAAC & 22 & 1.76 \\
\hline mmu-miR-24 & UGGCUCAGUUCAGCAGGAACAG & 22 & 1.46 \\
\hline mmu-miR-23b & AUCACAUUGCCAGGGAUUACC & 21 & 1.43 \\
\hline mmu-miR-497 & CAGCAGCACACUGUGGUUUGUA & 22 & 1.09 \\
\hline mmu-miR-28 & AAGGAGCUCACAGUCUAUUGAG & 22 & 1.06 \\
\hline mmu-miR-324-3p & CCACUGCCCCAGGUGCUGCU & 20 & 0.71 \\
\hline mmu-miR-363-3p & AAUUGCACGGUAUCCAUCUGUA & 22 & -0.76 \\
\hline mmu-miR-344c & UGAUCUAGUCAAAGCCUGACAGU & 23 & -0.77 \\
\hline mmu-miR-344d & GAUAUAACCACUGCCAGACUGA & 22 & -0.85 \\
\hline mmu-miR-216a & UAAUCUCAGCUGGCAACUGUGA & 22 & -1.39 \\
\hline mmu-miR-137 & UUAUUGCUUAAGAAUACGCGUAG & 23 & -1.44 \\
\hline mmu-miR-10a-3p & CAAAUUCGUAUCUAGGGGAAUA & 22 & -1.65 \\
\hline mmu-miR-10b-3p & CAGAUUCGAUUCUAGGGGAAUA & 22 & -1.92 \\
\hline mmu-miR-124-5p & CGUGUUCACAGCGGACCUUGAU & 22 & -2.19 \\
\hline mmu-miR-9 & UCUUUGGUUAUCUAGCUGUAUGA & 23 & -2.27 \\
\hline mmu-miR-7a-2-3p & CAACAAGUCCCAGUCUGCCACA & 22 & -2.84 \\
\hline mmu-miR-135a-2-3p & UGUAGGGAUGGAAGCCAUGAA & 21 & -3.02 \\
\hline mmu-miR-615-3p & UCCGAGCCUGGGUCUCCCUCUU & 22 & -3.22 \\
\hline mmu-miR-344 & UGAUCUAGCCAAAGCCUGACUGU & 23 & -3.39 \\
\hline mmu-miR-10b & UACCCUGUAGAACCGAAUUUGUG & 23 & -3.81 \\
\hline mmu-miR-219-3p & AGAAUUGUGGCUGGACAUCUGU & 22 & -3.94 \\
\hline mmu-miR-9-3p & AUAAAGCUAGAUAACCGAAAGU & 22 & -5.13 \\
\hline mmu-miR-10a & UACCCUGUAGAUCCGAAUUUGUG & 23 & -5.61 \\
\hline
\end{tabular}

nt: nucleotides 
mRNA expression

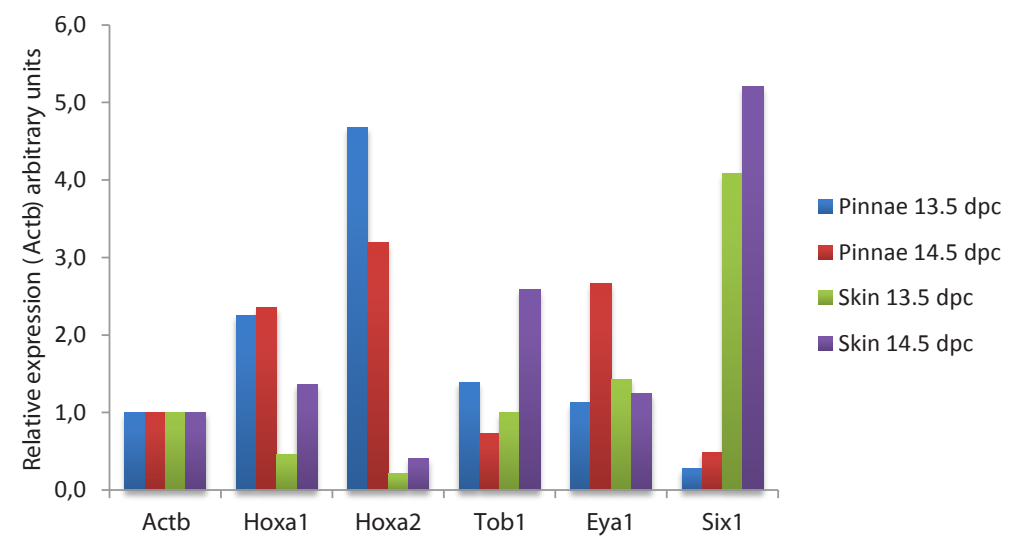

Fig. 4. mRNA relative expression levels at $\mathbf{1 3 . 5}$ and $\mathbf{1 4 . 5}$ dpc. Comparative $m R N A$ expression levels in pinnae and back skin tissues of putative mRNA targets of the mmu-miR studied. Relative expression levels for each gene and each comparison between tissues were calculated using the formula of relative expression ( $R=2^{-\Delta \Delta C p}$ ) (Pfaffl, 2006).

Runx2, Crim1 and Dicer1) that could be participating in external ear development in mice. Zeb1 and Zeb2 encode transcription factors that are involved in neural development and in the development of tissues derived from neural crest cells (Shin et al., 2012). Tob1 may negatively regulate the BMP/Smad signaling pathway in osteoblasts, a signaling pathway that has also been implicated in external ear development of early stage mice (Yoshida et al., 2000; Minoux et al., 2013) We found mRNA of Tob1 in pinnae (Fig. 4), but the interaction with mmu-miR-200c, mmu-miR-205 and mmu-miR-429, their possible regulators, is still unknown. The remaining genes, Sani1, Runx1 and Runx2, have been implicated in the process of chondrogenesis (Yoshida and Komori, 2005; Chen and Gridley, 2013).

In summary, we observed that there is a specific spatiotemporal pattern of miRNA expression during external ear development at $13.5 \mathrm{dpc}$ and $14.5 \mathrm{dpc}$.

This analysis is the first miRNA expression profiling study during external ear development in mammals. Thus, it could set up the basis for understanding the role of miRNAs in normal external ear development in mammals and for determining miRNA involvement in the etiology of external ear congenital malformations, such as microtia.

\section{Materials and Methods}

\section{Mice}

The CD1 mouse strain was used in this study. Scheduled mating was carried out in order to obtain fetuses at specific developmental stages. Pregnant females were sacrificed at $13.5 \mathrm{dpc}$ or $14.5 \mathrm{dpc}$. The gestational age of the fetus was determined by the day of appearance of the vaginal plug $(0.5 \mathrm{dpc})$ and confirmed by morphological criteria. All procedures were performed in accordance with the Mexican regulation NOM-062-ZOO-1999 norm and the Guide for the Care and Use of Laboratory Animals, Institute for Laboratory Animal Research of the National Academy of Sciences. The Institutional Animal Care and Use of Laboratory Animals and Research Committees from the Instituto Nacional de Pediatría approved this study.

\section{Tissues samples and RNA extraction}

Biological triplicates for each tissue and each development stage were analyzed. One litter represents one biological replica and each litter had an average of 16 fetuses. External ear tissue, the pinna (cartilage and skin), and back skin tissues were dissected under stereomicroscope in iced and cooled nuclease free PBS (Gibco, Waltham, MA USA) and were kept in RNAlater (Ambion, Waltham, MAUSA) at $-80^{\circ} \mathrm{C}$ until further processing. The region used as reference (back skin) is of an ectodermal origin, is close to the pinnae on the embryo and it differentiates at the same developmental stage. Using the reference region, we were able to identify the miRNAs that were specifically expressed in the external ear in these stages. Total RNA extraction including short RNAs (<200 bp) was performed with an RNA Mini elute kit (Qiagen, Venlo, Netherlands) and quantification was performed with a Nanodrop ${ }^{\mathrm{TM}}$ spectrophotometer (Nanodrop Technologies, Wilmington, DE, USA). RNA quality was monitored by agarose gel electrophoresis.

\section{miRNA array analysis}

Affymetrix GeneChip miRNA Arrays 3.0 (Affymetrix, Santa Clara, CA, USA) were used to screen the miRNA profile expression during external ear development. A single array is comprised of 179,217 probes that represent 19,913 mature miRNAcontained in miRBase V.17 (www.mirbase.org) with probes for 153 different organisms, including 1111 probes for Mus musculus miRNAs and 855 pre-miRNAs. The array analysis followed the manufacturer's instructions. Briefly, $500 \mathrm{ng}$ of total RNA, including short RNAs, was labeled with the FlashTag ${ }^{\circledR}$ Biotin HSR kit (Genisphere/Affymetrix, Santa Clara, CA, USA) and the labeling was tested using an Enzyme Linked Oligosorbent Assay (ELOSA) QC Assay (Thermo Fisher Scientific Inc, Waltham, MA, USA). The denaturalized samples were then injected into array chips and hybridized at $48^{\circ} \mathrm{C}$ and $60 \mathrm{rpm}$ for 17 hours in an Affymetrix Hybridization Oven 645. Arrays were then stained and washed in the Affymetrix GeneChip Fluidic Station 450 and scanned using an Affymetrix GeneChip Scanner 3000 7G (Affymetrix, Santa Clara, CA, USA).

The array data were normalized by global normalization using the miRNA QC Tool of the Expression Console software (Affymetrix, Santa Clara, CA, USA). To identify differentially expressed miRNAs at each stage, linear regression analysis using the Bioconductor LiMMa (Lineal Models for Microarrays) algorithm was employed. Hierarchical clustering analysis was performed with the Bioconductor and Transcriptome Analysis Console software from Affymetrix. The microarrays data were deposited in the Gene Expression Omnibus database (accession number GSE64945).

Putative mRNA targets for differentially expressed miRNAs were identified using the bioinformatic predicting tools TargetScanMouse Release 6.2 (www.targetscan.org) miRNAbody Map (www.mirnabodymap.org), miRBase (www.mirbase.org) and DIANA Tools (Tarbase, microT v4 and microT-CDS), (http://diana.imis.athena-innovation.gr/DianaTools).

\section{Real-time quantitative RT-PCR analysis}

In order to know if target mRNAs are present in pinnae or skin at the stages studied, we realize real-time quantitative RT-PCR analysis; 500 ng of total RNA was treated with $0.1 \mathrm{U}$ RNase-free DNase I (Invitrogen, Carlsbad, CA, USA) in $20 \mathrm{mM}$ Tris- $\mathrm{HCl}, \mathrm{pH} 8.3,50 \mathrm{mM} \mathrm{KCl}$, and $1 \mathrm{mM}$ $\mathrm{MgCl}_{2}$ for $15 \mathrm{~min}$ at room temperature, the enzyme was then inactivated by adding EDTA to a final concentration $1 \mathrm{mM}$ followed by incubation at $65^{\circ} \mathrm{C} / 10 \mathrm{~min}$. After this step total RNA was retro-transcribed using the Transcriptor First Strand cDNA Synthesis Kit (Roche Diagnostics, GmbH, Mannheim, Germany) using anchored-oligo (dT) 18 primer, 50-pmol/ul and Random hexamer primer, $600 \mathrm{pmol} / \mu \mathrm{L}$, Protector RNase Inhibitor 20 $\mathrm{U}$ and $10 \mathrm{U}$ of Transcriptor Reverse Transcriptase. The cDNA obtained was quantified using a Nanodrop ND 1000 spectrophotometer (Nanodrop 
TABLE 3

\section{USED TO DETECT MRNA EXPRESSION BY qRT-PCR}

\begin{tabular}{|c|c|c|}
\hline Gene & Primer sequence $5^{\prime}-3^{\prime}$ & Probe sequence $5^{\prime}-3^{\prime}$ \\
\hline Hoxa1 & $\begin{array}{l}\text { F: GCC CTG GCC ACG TAT AAT AA } \\
\text { R: GAA GCA GGG GAA CGA CAG }\end{array}$ & CAG CCA CC \\
\hline Hoxa2 & $\begin{array}{l}\text { F: ACC TGG AGG ACT CGG ACA A } \\
\text { R: GGG CTT GCT CAA AGA GTG AC }\end{array}$ & GGA GGA AG \\
\hline Tob1 & $\begin{array}{l}\text { F: ACT TTT GCT GCC ACC AAG TT } \\
\text { R: GAG CTA CCT TGC TGC TAC GG }\end{array}$ & CTC CAC CA \\
\hline Six1 & $\begin{array}{l}\text { F: GGT CTG CAA GCC CAT CAG } \\
\text { R: AGT CCA CCA AAC TGG AGG TG }\end{array}$ & GCT CCA GG \\
\hline Eya1 & $\begin{array}{l}\text { F: CGA GGT TCA GAT GGG AAG TC } \\
\text { R: GGG AGG GGA GGG ATT ATT G }\end{array}$ & GGC AGA AG \\
\hline Actb & $\begin{array}{l}\text { F: GAT CAT TGC TCC TCC TGA GC } \\
\text { R: CCG GAC TCA TCG TAC TCC TG }\end{array}$ & CTC CAC CA \\
\hline
\end{tabular}

Technologies, Wilmington, DE, USA).

Real-time quantitative-PCR (q-PCR) was performed on cDNA samples in duplicate for two biological repeats. The q-PCR was performed with the Universal Probes system (Roche Diagnostics, GmbH, Mannheim, Germany) and the Light Cycler Taq Man Master kit (Roche Diagnostics, GmbH, Mannheim, Germany). Besides target genes expression, Actb expression was quantified as reference gene. Primers for each gene were designed on line with the ProbeFinder Software (www.universalprobelibrary.com) and manufactured at Sequencing and Synthesis Unit (IBT, UNAM). The primers and the universal probe sequences for each gene are indicated in Table 3.

The q-PCR was carried out in the Light Cycler 2.0 Carousel Roche equipment and the conditions were as follows: 1 cycle at $95^{\circ} \mathrm{C}$ for $10 \mathrm{~min}$ utes, denaturalization at $95^{\circ} \mathrm{C}$ for 10 seconds, annealing at $60^{\circ} \mathrm{C}$ for 30 seconds and extension and detection at $72^{\circ} \mathrm{C}$ for 5 seconds for a total of 45 cycles. Relative expression levels for each gene and for each tissue were calculated using the formula of relative expression $R=2^{-\Delta \Delta C p}$ (Pfaffl, 2006).

\section{Whole mount in situ hybridization}

The protocol followed was the recommended by Exiqon (Exiqon, Vedbæk, Denmark) (Thisse et al., 2004; Kloosterman et al., 2006) with modifications. Briefly, embryos were fixed overnight at $4{ }^{\circ} \mathrm{C}$ in $4 \%$ paraformaldehyde in PBS and subsequently transferred to $25 \%, 50 \%, 75 \%$ and $100 \%$ methanol in PBST (PBS containing $0.1 \%$ Tween-20), and stored at $-20^{\circ} \mathrm{C}$ until further processing. At the first day of the in situ hybridization embryos were rehydrated by successive washes for $5 \mathrm{~min}$ at room temperature (RT) in $75 \%, 50 \%, 25 \%$ and $100 \%$ PBST ( $4 \times 5$ min). Embryos were treated with $10 \mathrm{ug} / \mathrm{ml}$ proteinase K (Roche Diagnostics, GmbH, Mannheim, Germany) in PBST for $45 \mathrm{~min}$ at $37^{\circ} \mathrm{C}$, refixed for $20 \mathrm{~min}$ in $4 \%$ paraformaldehyde in PBS at RT and washed $5 \times 5$ min with PBST at RT. The embryos were then transferred to hybridization mix $(\mathrm{HM})(50 \%$ Formamide, $5 x \mathrm{SSC}, \mathrm{pH}$ 6.0, $0.1 \%$ Tween, $9.2 \mathrm{mM}$ citric acid, $50 \mathrm{ug} / \mathrm{ml}$ heparin, $500 \mathrm{ug} / \mathrm{ml}$ yeast RNA) and prehybridized for $2-3$ hour at the hybridization temperature $\left(57^{\circ} \mathrm{C}\right.$ for probes mmu-miR-205 and mmu-miR-200c, $53^{\circ} \mathrm{C}$ for probe mmu-miR10a). Hybridization was performed in fresh pre-heated hybridization buffer containing $10 \mathrm{nM}$ of DIG labeled LNA probe (Exiqon, Vedbæk, Denmark) overnight, at the hybridization temperature. Post-hybridization washes were done at the hybridization temperature by successive incubations for 15 min in HM without heparin and yeast RNA, 75\% HM-/25\% 2x SSC, 50\% $\mathrm{HM}-/ 50 \% 2 \mathrm{xSC}, 25 \% \mathrm{HM}-/ 75 \%$ 2x SSC, $100 \%$ 2x SSC, twice in $0.2 x$ SSC 30 min. Subsequently, embryos were transferred to PBST through successive incubations for $10 \mathrm{~min}$ at RT in $75 \% 0.2 \times$ SSC/25\% PBST, $50 \%$ 0.2x SSC/50\% PBST, $25 \%$ 0.2x SSC/75\% PBST and $100 \%$ PBST. After washing the embryos were exposed for 1 hour in blocking buffer (1X blocking in PBST) (Roche Diagnostics, $\mathrm{GmbH}$, Mannheim, Germany), then were incubated overnight at $4^{\circ} \mathrm{C}$ in blocking buffer containing anti-DIG-AP FAB fragments (1:2000) (Roche Diagnostics, GmbH, Mannheim, Germany). The next day, embryos were washed once in PBST 5 min RT, after $5 \times 1$ hour in PBST and then at $4^{\circ} \mathrm{C}$ in PBST overnight. After the post-antibody washes, the embryos were washed $3 \times 5 \mathrm{~min}$ in staining buffer $(100 \mathrm{mM}$ Tris $\mathrm{HCl} \mathrm{pH9.5,} 50 \mathrm{mM} \mathrm{MgCl}, 100 \mathrm{mM} \mathrm{NaCl}, 0.1 \%$ tween 20). Staining was done in buffer supplied with $4.5 \mathrm{ul} / \mathrm{ml}$ NBT $(50 \mathrm{mg} / \mathrm{ml}$ stock)(Roche Diagnostics, GmbH, Mannheim, Germany) and $3.5 \mathrm{ul} / \mathrm{ml} \mathrm{BCIP}(50 \mathrm{mg} / \mathrm{ml}$ stock)(Roche Diagnostics, GmbH, Mannheim, Germany). The reaction was stopped with $1 \mathrm{mM}$ EDTA in PBST and the embryos were refixed for $60 \mathrm{~min}$ in 4\% paraformaldehyde in PBS at RT and washed $5 \times 5$ min with PBST at RT. For observation and imaging the embryos were mounted in $50 \%$ glycerol. The embryos were observed under a stereoscopic microscope over petri dishes coated with a layer of $1 \%$ agarose $( \pm 2 \mathrm{~mm}$ thick) prepared in PBS; pictures were taken using a digital camera, first for the complete embryo and then for specific structures as pinnae and limbs.

\section{Acknowledgments}

Financial support: Federal Funding from the Instituto Nacional de Pediatría 053/2013. U. Juarez received a CONACyT scholarship 270077. We would like to thank the personnel of the animal care facility of the Instituto Nacional de Pediatría, for their professional work with the mice, to Dra. Diana Escalante for their valuable advice for in situ hybridization technique and to Dra. Ana Valeria Martínez Silva from Instituto de Fisiología Celular, UNAM for technical support, to Dr. Benilde García de Teresa for their valuable comments and revision of the manuscript, to Dr. Pablo González for bioinformatics analysis, and to the Sequencing and Synthesis Unit from the Instituto de Biotecnología, UNAM, for primer synthesis.

\section{References}

BEREZIKOV E (2011). Evolution of microRNA diversity and regulation in animals Nat Rev Genet 12: 846-860.

CHEN Y, GRIDLEY T (2013). Compensatory regulation of the Snai1 and Snai2 genes during chondrogenesis. J Bone Miner Res 28: 1412-1421.

GARZON R, PICHIORRI F, PALUMBO T, IULIANO R, CIMMINO A, AQEILAN R, VOLINIAS, BHATT D, ALDER H, MARCUCCI G, CALIN GA, LIU CG, BLOOMFIELD CD, ANDREEFF M, CROCE CM (2006). MicroRNA fingerprints during human megakaryocytopoiesis. Proc Natl Acad Sci USA 103: 5078-5083.

GAVALAS A, STUDER M, LUMSDEN A, RIJLI FM, KRUMLAUF R, CHAMBON P (1998). Hoxa1 and Hoxb1 synergize in patterning the hindbrain, cranial nerves and second pharyngeal arch. Development 125: 1123-1136.

JONES FW, CHUAN WI (1934). The development of the external ear. J. Anat 68:525-533

KALATZIS V, SAHLY I, EL-AMRAOUI A, PETIT C (1998). Eya1 expression in the developing ear and kidney: towards the understanding of the pathogenesis of Branchio-Oto-Renal (BOR) syndrome. Dev Dyn 213: 486-99

KLOOSTERMAN WP, WIENHOLDS E, de BRUJIN E, KAUPPINNEN S, PLASTERK $\mathrm{RH}$ (2006). In situ detection of miRNAs in animal embryos using LNA-modified oligonucleotide probes. Nat Methods 3: 27-29

LAU NC, LIM LP, WEINSTEINEG, BARTELDP (2001). An abundant class of tiny RNAs with probable regulatory roles in Caenorhabditis elegans. Science 294: 858-862.

LI C, HAO S, WANG H, JIN L, QING F, ZHENG F, ZHANG P, CHEN L, MA D, ZHANG $T$ (2013). MicroRNA expression profiling and target genes study in congenital microtia. Int J of Pediatr Otorhinolaryngol 77: 483-487

LUQUETTI DV, HEIKE CL, HING AV, CUNNINGHAM ML, COX TC (2012). Microtia Epidemiology and genetics. Am J Med Genet 158A: 24-139.

MALLO M, GRIDLEY T (1996). Development of the mammalian ear: Coordinate regulation of formation of the tympanic ring and the external acoustic meatus Development 122: 173-179.

MINOUX M, KRATOCHWIL CF, DUCRET S, AMIN S, KITAZAWA T, KURIHARA H, BOBOLAN, VILAIN N, RIJLI FM (2013). Mouse Hoxa2 mutations provide a model for microtia and auricle duplication. Development 140: 4386-4397.

NEO WH, YAP K, LEE SH, LOOI LS, KHANDELIA P, NEO SX, MAKEYEV EV, SU IH (2014). MicroRNA miR-124 controls the choice between neuronal and astrocyte differentiation by fine-tuning Ezh2 expression. J Biol Chem 289: 20788-20801.

PASQUINELLI AE (2012). Non-Coding RNAMicroRNAs and their targets: recognition, regulation and an emerging reciprocal relationship. Nat Rev Genet 13: 271-282.

PETRI R, MALMEVIK J, FASCHING L, AKERBLOM M, JAKOBSSON J (2014) 
miRNAs in brain development. Exp Cell Res 321: 84-89.

PFAFFL MW (2006). Relative quantification. In Real-time PCR. (Ed. M. Tevfik Dorak), Taylor \& Francis Group. New York, N Y. pp 63-80.

SACHELI R, NGUYEN L, BORGS L, VANDENBOSCH R, BODSON M, LEFEBVRE P, MALGRANGE P (2009). Expression patterns of miR-96, miR-182 and miR-183 in the developing inner ear. Gene Expr Patterns 9:364-370.

SANUKI R, ONISHI A, KOIKE C, MURAMATSU R, WATANABE S, MURANISHI Y, IRIE S, UNEO S, KOYASU T, MATSUI R (2011). miR-124a is required for hippocampal axogenesis and retinal cone survival through $L h x 2$ suppression. Nat Neurosci 14: 1125-1134.

SHIN JO, KIM EJ, CHO KW, NAKAGAWA E, KWON HJ, CHO SW, JUNG HS (2012). BMP4 signaling mediates Zeb family in developing mouse tooth. Histochem Cell Biol 137: 791-800.

SONG MH, KWONTJ, KIM HR, JEON JH, BAEKJI, LEE WS, KIM UK, CHOIJY (2013). Mutational analysis of EYA1, SIX1 and SIX5 genes and strategies for management of hearing loss in patients with BOR/BO syndrome. PLoS One 8: e67236
TANZER A, AMEMIYA CT, KIM CB, STADLER PF (2005). Evolution of microRNAs located within Hox gene clusters. J Exp Zool B Mol Dev Evol 304: 75-85.

YOSHIDA CA, KOMORI T (2005). Role of Runx proteins in chondrogenesis. Crit Rev Eukaryot Gene Expr 15: 243-254.

THISSE B, HEYER V, LUX A, ALUNNI V, DEGRAVE A, SEILIEZ I, KIRCHNER J, PARKHILL JP, THISSE C. (2004). Spatial and temporal expression of the zebrafish genome by large-scale in situ hybridization screening. Methods Cell Biol 77: 505-519.

WARNER DR, MUKHOPADHYAY P, BROCK G, WEBB CL, PISANO MM, GREENE RM (2014). MicroRNA expression profiling of the developing murine upper lip. Develop Growth Differ 56: 434-447.

YOSHIDAY, TANAKAS, UMEMORI H, MINOWAO, USUI M, IKEMATSU N, HOSODA E, IMAMURA T, KUNO J, YAMASHITA T, MIYAZONO K, NODA M, NODA T, YAMAMOTO T (2000). Negative regulation of BMP/Smad signaling by Tob in osteoblasts. Cell 103: 1085-1097.

ZHENG W, HUANG L, WEI ZB, SILVIUS D, TANG B, XU PX (2003). The role of Six1 in mammalian auditory system development. Development 130: 3989-4000. 


\section{Further Related Reading, published previously in the Int. J. Dev. Biol.}

The molecular biology of ear development - "Twenty years are nothing" Fernando Giraldez and Bernd Fritzsch Int. J. Dev. Biol. (2007) 51: 429-438

http://www.intjdevbiol.com/web/paper/072390fg

Pocket proteins and cell cycle regulation in inner ear development Sonia M.S. Rocha-Sanchez and Kirk W. Beisel Int. J. Dev. Biol. (2007) 51: 585-595

http://www.intjdevbiol.com/web/paper/072387sr

Patterning and morphogenesis of the vertebrate inner ear Jinwoong Bok, Weise Chang and Doris K. Wu Int. J. Dev. Biol. (2007) 51: 521-533

http://www.intjdevbiol.com/web/paper/072381jb

Molecular evolution of the vertebrate mechanosensory cell and ear Bernd Fritzsch, Kirk W. Beisel, Sarah Pauley and Garrett Soukup Int. J. Dev. Biol. (2007) 51: 663-678 http://www.intjdevbiol.com/web/paper/072367bf

Mouse models to study inner ear development and hereditary hearing loss Lilach M. Friedman, Amiel A. Dror and Karen B. Avraham Int. J. Dev. Biol. (2007) 51: 609-631 http://www.intjdevbiol.com/web/paper/072365lf

5 yr ISI Impact Factor $(2013)=2.879$
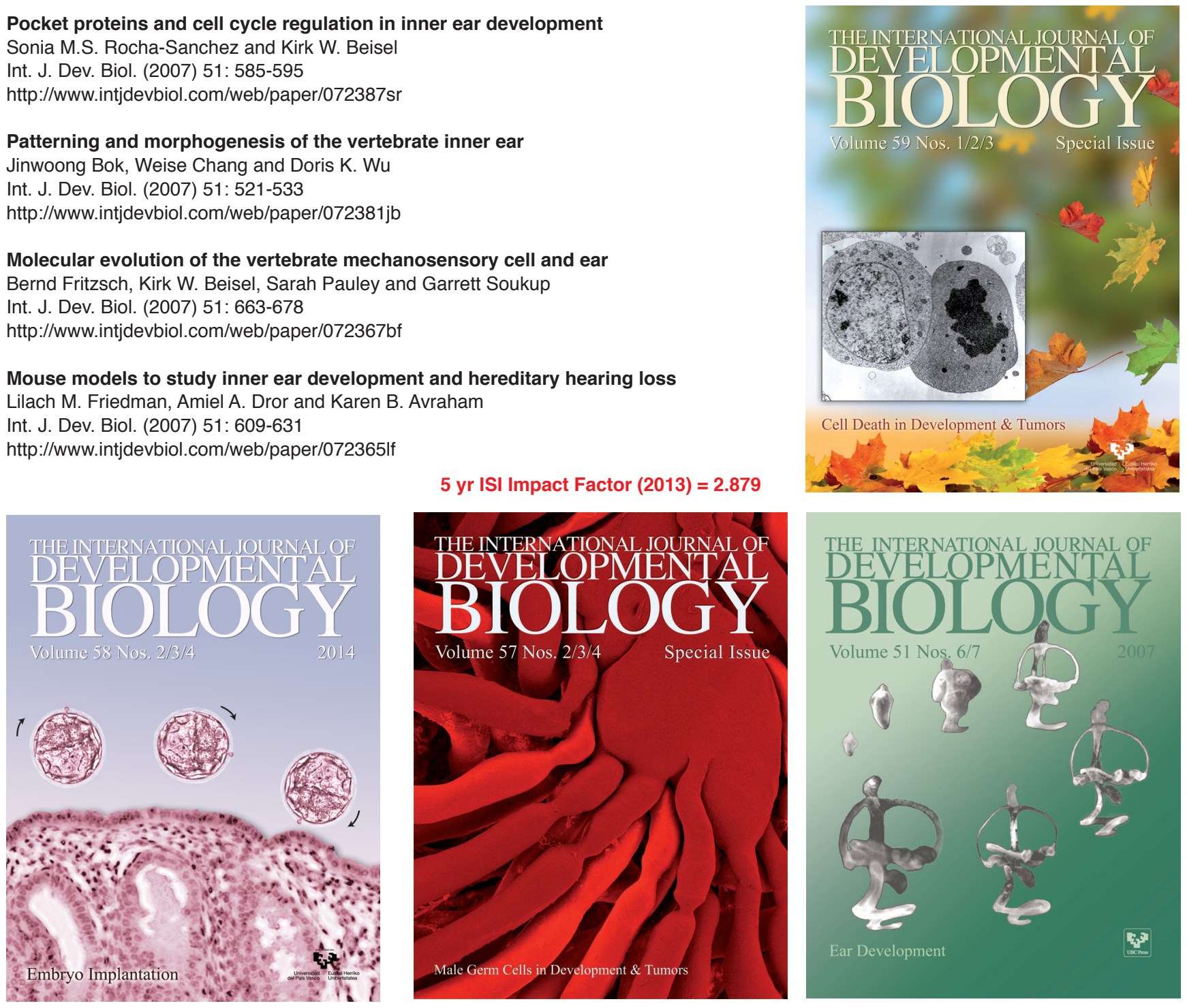